\title{
Impulsivity in rodents with a genetic predisposition for excessive alcohol consumption is associated with a lack of a prospective strategy
}

\author{
David N. Linsenbardt ${ }^{1}$ • Michael P. Smoker ${ }^{1}$ • Sarine S. Janetsian-Fritz ${ }^{1}$. \\ Christopher C. Lapish ${ }^{1,2,3,4}$
}

Published online: 20 December 2016

(C) Psychonomic Society, Inc. 2016

\begin{abstract}
Increasing evidence supports the hypothesis that impulsive decision-making is a heritable risk factor for an alcohol use disorder (AUD). Clearly identifying a link between impulsivity and AUD risk, however, is complicated by the fact that both AUDs and impulsivity are heterogeneous constructs. Understanding the link between the two requires identifying the underlying cognitive factors that lead to impulsive choices. Rodent models have established that a family history of excessive drinking can lead to the expression of a transgenerational impulsive phenotype, suggesting heritable alterations in the decision-making process. In the present study, we explored the cognitive processes underlying impulsive choice in a validated, selectively bred rodent model of excessive drinking - the alcohol-preferring ("P") rat. Impulsivity was measured via delay discounting (DD), and $P$ rats exhibited an impulsive phenotype as compared to their outbred foundation strain-Wistar rats. Steeper discounting in $\mathrm{P}$ rats was associated with a lack of a prospective behavioral strategy, which was observed in Wistar rats and was directly related to DD. To further explore the underlying cognitive
\end{abstract}

David N. Linsenbardt

dlinsenb@iupui.edu

$1 \quad$ Addiction Neuroscience, Department of Psychology, Indiana University-Purdue University Indianapolis, 402 N. Blackford Street, LD 124, Indianapolis, IN 46202, USA

2 Stark Neuroscience Institute and Indiana Alcohol Research Center, Indiana University School of Medicine, Indianapolis, IN, USA

3 Institute for Mathematical Modeling and Computational Sciences, Indiana University-Purdue University Indianapolis, Indianapolis, IN, USA

4 Indiana Alcohol Research Center, Indiana University School Of Medicine, Indianapolis, IN 46202, USA factors mediating these observations, a drift diffusion model of DD was constructed. These simulations supported the hypothesis that prospective memory of the delayed reward guided choice decisions, slowed discounting, and optimized the fit of the model to the experimental data. Collectively, these data suggest that a deficit in forming or maintaining a prospective behavioral plan is a critical intermediary to delaying reward, and by extension, may underlie the inability to delay reward in those with increased AUD risk.

Keywords Delay discounting · Alcoholism . Alcohol-preferring rat $\cdot$ Computational model $\cdot$ Prospective memory $\cdot$ Drift diffusion model

Impulsivity is broadly defined as the predisposition to act prematurely without foresight (Dalley, Everitt, \& Robbins, 2011). The propensity to make impulsive choices is a risk factor for substance abuse disorders including alcoholism; however, the strength of this relationship is unknown (de Wit, 2009; Dick et al., 2010; Dougherty et al., 2014; Jentsch et al., 2014; Jentsch \& Pennington, 2014; Lejuez et al., 2010; MacKillop, 2013; Verdejo-Garcia, Lawrence, \& Clark, 2008). One challenge in accurately quantifying this relationship is that both impulsivity and alcohol use disorders (AUDs) are heterogeneous constructs, influenced by a number of factors, such as sensation seeking, positive/negative urgency, or a lack of planning (Coskunpinar, Dir, \& Cyders, 2013; Dalley et al., 2011; de Wit, 2009; Jentsch et al., 2014; Lejuez et al., 2010; Rogers, Moeller, Swann, \& Clark, 2010). The broad goal of the present study was to explore the underlying factors contributing to the relationship between impulsivity and risk for excessive drinking.

Delay discounting (DD) is a behavioral measure of cognitive impulsivity, and a relationship has been reported between DD and AUD (Bickel, Johnson, Koffarnus, MacKillop, \& 
Murphy, 2014; Bickel, Koffarnus, Moody, \& Wilson, 2014; Jentsch et al., 2014; Kollins, 2003; MacKillop, 2013; MacKillop et al., 2011; Mitchell, 2011). Both alcoholism risk (Dick \& Bierut, 2006; Enoch \& Goldman, 2001; Johnson, van den Bree, Gupman, \& Pickens, 1998) and DD (Anokhin, Grant, Mulligan, \& Heath, 2014) are heritable, and there is increasing evidence that these traits are also genetically correlated (Acheson, Richard, Mathias, \& Dougherty, 2011; Dougherty et al., 2014; Oberlin \& Grahame, 2009; Perkel, Bentzley, Andrzejewski, \& Martinetti, 2015; Wilhelm \& Mitchell, 2008). It is still unclear, however, to what extent excessive DD increases risk of developing an AUD, and which underlying cognitive processes might mediate this risk.

Alcohol-preferring "P" rats have been selectively bred for excessive alcohol consumption and have proven extremely useful for exploring the heritable factors and neural systems leading to this phenotype (McBride, Rodd, Bell, Lumeng, \& $\mathrm{Li}, 2014)$. Evidence is emerging that, like humans with a family history of alcoholism, rodents selectively bred for excessive drinking also exhibit impaired measures of cognition-including impulsivity (Beckwith \& Czachowski, 2016; Oberlin \& Grahame, 2009; Walker, Pena-Oliver, \& Stephens, 2011; Wenger \& Hall, 2010). Moreover, alcohol-naive P rats discount delayed rewards more robustly than do rodent populations that lack increased genetic risk (Beckwith \& Czachowski, 2014; Perkel et al., 2015). These data suggest that the impulsive phenotype is not a consequence of excessive drinking, but rather that these two traits are regulated by similar genes and neural systems. The specific goal of the present study was to further characterize how altered cognitive processing in $\mathrm{P}$ rats predisposes them toward impulsive decision-making.

Drift diffusion models provide a powerful tool to explore the cognitive processes guiding fast, two-choice decisions (Ratcliff \& McKoon, 2008). These models have been used extensively to examine cognitive processes required to withhold a prepotent response (Verbruggen \& Logan, 2009), in addition to how environmental stimuli guide perceptualbased decisions (Romo \& Salinas, 2001; Shadlen, Britten, Newsome, \& Movshon, 1996). Two-choice perceptual decision-making modeled as a diffusion process assumes that noisy sensory information is integrated to guide decisionmaking, and can account for the shape of response time distributions, the speed-accuracy trade-off, and changes in neural firing during intertemporal choices (Kim \& Lee, 2011; Ratcliff \& McKoon, 2008). A recent study using a drift diffusion model supported the hypothesis that assigning value to a delayed reward requires that it be mapped to the context in which it will be received (Kurth-Nelson, Bickel, \& Redish, 2012). These simulations concluded that the mapping process requires prospective memory and is critical for delaying reward (Kurth-Nelson et al., 2012). The drift diffusion model presented herein also explores how future, delayed rewards, are mapped and maintained during cognitive search, and was used to further test the hypothesis that steep DD in P rats is due in part to alterations in the use of a prospective strategy. This was motivated by the fact that of the candidate cognitive processes that might mediate DD to increase AUD risk, the ability to, or use of, envisioning the future (i.e., prospection) has been shown recently to be negatively related to the severity of an individuals drinking problems (Griffiths et al., 2012) or alcohol dependence (Heffernan, 2008). Using a modified DD task in rodent populations with varying risk of excessive alcohol consumption, and a drift diffusion model of these data, these studies tested the hypothesis that the ability to plan goaldirected behaviors involves a cognitive search process that is influenced by genetic risk and mediates impulsivity.

\section{Method}

\section{Animals}

Sixteen male Indiana University P rats and 16 non-geneticallypredisposed (heterogeneous) Wistar rats were provided by the Indiana University Alcohol Research Center animal production core or purchased from Harlan (Indianapolis, IN), respectively. Upon arrival animals were acclimated to single housing and a 12-h reverse light/dark cycle with lights off at 7:00 AM for at least a week prior to testing. All animals were at least 70 days of age prior to testing and had $a d l i b$ access to food and water prior to food restriction/habituation. Over the course of approximately two weeks, animals were food restricted to $85 \%$ of their starting free-feeding weight and maintained under this condition throughout all experiments. All procedures were approved by the Purdue School of Science Animal Care and Use Committee and conformed to the Guidelines for the Care and Use of Mammals in Neuroscience and Behavioral Research (National Research Council, 2003).

\section{Operant apparatus}

Standard one compartment operant boxes $(20.3 \mathrm{~cm} \times 15.9 \mathrm{~cm}$ $\times 21.3 \mathrm{~cm}$; Med Associates, St Albans, VT) were used for all DD procedures. Each box contained left and right retractable levers on one wall, left and right stimulus lights positioned immediately above each lever, and an easily accessible pellet hopper positioned between these left and right positioned devices. The opposite wall contained a house light and a tone generator $(2900 \mathrm{~Hz})$ on the topmost position.

\section{Habituation/shaping}

Ten sucrose pellets were placed in the animals' home cage on the final day of the two-week induction/maintenance of food deprivation. Animals were then habituated to the operant apparatus and trained to press levers for the delivery 
of sucrose pellets. The first day served to habituate the animals to the operant box and familiarize them with the location of the pellet hopper in which sucrose pellets were delivered. Animals were placed in the operant box for 30 min during which ten sucrose pellets were already available in the hopper. Animals were then progressively shaped to press the levers for delivery of sucrose pellets and habituated to the stimuli and stimuli contingencies over approximately eight consecutive days. On Day 1 of shaping, single sucrose pellets were delivered into the food hopper with increasing time intervals over a 20 minute period (30 total trials/pellets); no stimuli were used other than the sucrose pellet dispenser. On Day 2 of shaping, illumination of the house light signaled the start of a trial. After $10 \mathrm{~s}$, it was extinguished and a single sucrose pellet was dispensed. The intertrial interval (ITI) on these sessions were 15,25 , or $35 \mathrm{~s}$, chosen at random (30 trials/ pellets total). The levers and the stimulus lights above the levers were introduced on Days 3 and 4, and animals were trained (i.e., "hand-shaped") to press the left and the right bars for sucrose pellet delivery using successive approximation. Animals were randomly assigned to one of two levers on the first day and the opposite lever was used the following day. Some animals required one additional day of training/shaping before moving on to the next training/shaping phase. A minimum of 30 leverpresses was required before moving on. On Days 5 and 6, illumination of the house light signaled the start of a trial. After $10 \mathrm{~s}$, it was extinguished and a single lever and corresponding stimulus light were extended and illuminated, respectively. A response on the lever resulted in the simultaneous sounding of a 100-ms tone (marking the reinforced response) and delivery of a single sucrose pellet. One lever/light side was used per day, and side assignments matched those on Days 3 and 4. Each session terminated following $30 \mathrm{~min}$ or 30 sucrose pellets earned, whichever came first. Days 7 and 8 included all of the stimuli. Illumination of the house light signaled the start of a trial. After $10 \mathrm{~s}$, the house light was extinguished and both levers were extended into the chamber. The animals were required to initiate the start of the trial by pressing any one of the two levers. If a lever was not pressed after $10 \mathrm{~s}$, the levers were retracted and the house light was presented again. If a trial was initiated, the levers retracted for $1 \mathrm{~s}$, and then were extended back into the chamber as choice levers with their corresponding stimulus lights illuminated. A response on either lever resulted in the simultaneous sounding of a 100-ms tone (marking the reinforced response) and delivery of a single sucrose pellet. Additionally, the stimulus light above the non-chosen bar was turned off while the light above the chosen lever remained on until the end of the trial. Trials were always $35 \mathrm{~s}$ in total duration. These sessions lasted $35 \mathrm{~min}$ or were terminated following 30 sucrose pellets earned. The preferred choice lever was determined for each animal from these final two sessions.

\section{Delay discounting}

Our within-session adjusting-amount DD procedures were adapted from Oberlin and Grahame (2009), and are illustrated in Table 1. The presentation of stimuli was identical to that during the last two days of shaping, except that lever pressing now resulted in the outcomes detailed here. For each animal, a "delay lever" was assigned to be the lever corresponding to the animal's nonpreferred side. Pressing the delay lever always resulted in the delivery of six sucrose pellets following a delay. The "immediate lever" was the opposite lever. Pressing the immediate lever resulted in the delivery of zero to six pellets immediately. The number of pellets delivered following a response on the immediate lever (i.e., the "value" of the immediate lever) always started at three on a given day. On "choice trials," the value of the immediate lever was increased by one pellet after each delay-lever press, and decreased by one pellet after each immediate-lever press. "Forced trials" were incorporated to expose the animals to both the delayed and immediate reinforcers. Forced trials resulted from a response on the same choice lever for two consecutive choice trials, and were presented on the following trial. Failure to respond on a forced trial resulted in the repe-

Table 1 Within-session, adjusting-amount delay-discounting procedures, adapted from those of Oberlin and Grahame (2009)

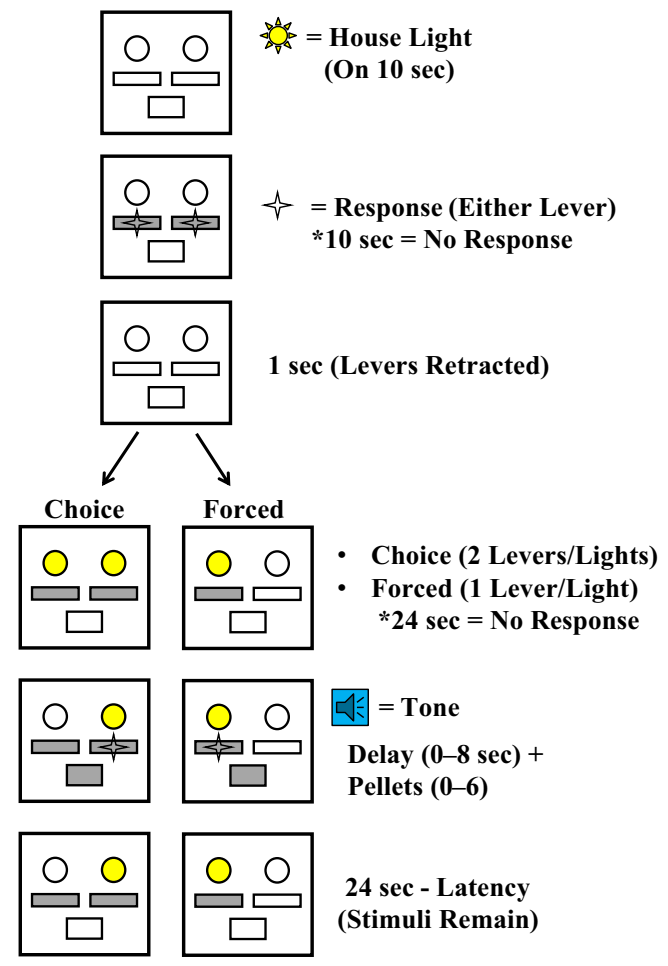


tition of an identical trial. Forced-trial responses did not affect the immediate-lever value. Each session terminated following 35 min or 30 choice trials completed. Four to six sessions were given for each delay in ascending, fixed delay intervals $(0,1$, $2,4,8$, and $16 \mathrm{~s}$ ). Animals were given six daily sessions at the 0 -s delay, and four daily sessions at every other delay. However, only animals who met our inclusion criteria of $70 \%$ of the maximum reward value of 6 (4.2 pellets) at the 0 -s delay were moved on to the other delays. The average value of the immediate lever over the last ten choice trials was determined for the last three days of each delay and was used to determine the indifference point of each animal. The mean indifference points for each delay were determined for all animals and evaluated as detailed below.

\section{Interlever response interval manipulation}

To better characterize choice lever consistency (see Results), a subset of the original Wistar rats were used for an additional follow-up experiment $(n=8)$. These studies were identical to the DD procedures detailed above, except that the latency with which the levers were re-extended following trial initiation presses was manipulated (i.e., the interlever response interval [IRI]). Animals were run for six consecutive days at each of three delays $(0,4$, and $16 \mathrm{~s})$. However, the IRI was increased over daily sessions (within each reward delay condition) from 1 to $16 \mathrm{~s}$. Animals first had two days at the 1-s (normal) IRI delay for each of the three reward delay conditions; one day to reacclimate them to the delay and the other for evaluating baseline behavior. For each subsequent day within a reward delay, IRI increased daily with delays of 2, 4, 8, and $16 \mathrm{~s}$. In total, animals had six consecutive days within three reward delays (18 days). Our hypothesis was that by increasing IRI, Wistar rats would shift away from using a consistent lever press strategy.

\section{Behavioral statistics}

The DD data were evaluated using methods adapted from Oberlin and Grahame (2009). We opted to use this model versus a generalized hyperbola model (Green \& Myerson, 2004) since it is simpler and reduces the number of variables necessary for the model. Mean indifference point across the final three days of each delay were evaluated using mixed factor ANOVAs, with rat population as the between-groups factor and delay as the within-groups factor. The rate of discounting was determined using the hyperbolic fitting function: $v=a /(1+k d)$ (Mazur, 1987). Here, $v$ is the subjective value of the reward, $a$ is the fixed value of the delay reward ( 6 pellets), $d$ is the length of the delay (0-16 s), and $k$ is the value fitted by the hyperbolic function. With this formula, impulsivity $(k)$ is measured by the steepness of the rate of discounting. Thus, the larger the $k$ value, the more impulsive the individual. Correlation and regression analyses were conducted to determine the strength and significance of particular variables of interest. Bonferroni corrected post-hoc tests were performed on the basis of the number of comparisons when appropriate. Data were considered significant at $p<.05$.

\section{Model of DD}

To assess the possible cognitive processes that guide decision making in the within-session adjusting-amount discounting task, a one-dimensional drift diffusion model was used to simulate this task. The model was constructed such that a random walk accumulated toward one of two possible "decision boundaries". The model was parameterized so one of the decision boundaries was found on each walk (i.e., as in the behavioral data, there were very few model omissions $[\leq 1 \%$; average experimental daily omissions: $P=0.5177 \pm$ 0.04 , Wistar $=0.42 \pm 0.08$ ). A trial was defined as a series of walks, with the location of the lower boundary corresponding to delay choices and the location of the upper boundary corresponding to immediate choices. The distance of the delay boundary from the origin was fixed at a distance that was proportional to the duration of the delay. The upper/ immediate boundary was adjusted on each walk (moved closer to or farther from the origin), with the distance from the origin inversely proportional to the subjective value $(v)$ of the reward. The immediate boundary was adjusted depending on which boundary had been found on the previous walk (i.e., the preceding "decision"), such that if a delay boundary had been found on the previous walk, the immediate boundary moved closer to the origin, and vice versa. Thus, conceptually, the immediate boundary distance represented the value of the immediate lever from behavioral experiments. One trial in the model consisted of 500 walks, which was meant to simulate one day of discounting at a fixed delay as in the behavioral experiments. One hundred trials (i.e., bootstraps) were run at each delay, which can be thought of as multiple observations (i.e., animals) over the delays. The step size and direction of the random walk $y$ was determined by

$y=\sum_{i=2}^{n}\left(d x_{i}\right)$

where $n$ was the duration of the random walk (5,000 steps for all simulations), and $d$ was a diffusion coefficient that adjusted the size of each step on the walk, which was set to 0.04 for all simulations. $x_{i}$ was randomly selected for each step $i$ from $\mathrm{N}(0,1)$, unless noted otherwise. The trajectory of the walk 
accumulated from the origin $\left(x_{1}=0\right)$, unless otherwise noted, toward a decision boundary located at either $1 / v$ or $-1 / v$, where $v$ was the subjective value of the reward defined by the discounting function described in the behavioral experiments:

$v=\frac{a}{1+k_{\langle e\rangle} d}$

where $a$ was equal to the actual value of the reward (in food pellets: [1 ...6]) and $d$ was equal to the temporal delay (in seconds; $[0,1,2,4,8,16])$. The DD parameter was defined as $k_{\langle e\rangle}$ (where $e$ denotes the mean $k$ from the experimental data; see the Results section). In this way, the decision boundaries $( \pm 1$ / v) representing higher-value "rewards" were set closer to the origin. Our rationale for this was based on the observation that high-value rewards have a higher probability of being chosen, and thus should be easier to locate in the "cognitive search space" (Howard, Fotedar, Datey, \& Hasselmo, 2005; Kurth-Nelson et al., 2012; Ratcliff \& McKoon, 2008).

The lower decision boundary was constructed as a proxy for a delayed choice, $a=6$, and was fixed so that $-1 / v$ was a fixed distance from the origin on each trial for a delay $(d)$. The upper decision boundary was designed as a proxy for an immediate choice, if the random walk crossed the upper decision boundary first (i.e., the immediate choice), the boundary was moved away from the origin on the next walk:

if $y=1 / v,\left\{\begin{array}{c}a \rightarrow a-1 \\ a<1, a \rightarrow 1\end{array}\right.$

However, if the trajectory crossed the lower decision boundary first (i.e., the delay choice), it was moved toward the origin:

if $y=-1 / v,\left\{\begin{array}{c}a \rightarrow a+1 \\ a>6, a \rightarrow 6\end{array}\right.$

To extract $k_{m}$ from each simulation (with $m$ denoting the modeled data), the mean of the immediate decision boundary was computed from each trial and for each bootstrap, which resulted in a Bootstrap $\times$ Delay matrix of values, and $k_{m}$ was then computed for each row, resulting in 100 values per simulation. The mean of these values is denoted as $k_{\langle m\rangle}$.

The origin of the random walk was adjusted in a subset of experiments to include a "memory" of which decision boundary had been found by the walk on the previous trial. The rationale for this feature was arrived at following initial simulations and was based on the premise that the accumulation of information about past choices, and how they might relate to the current choice, is a necessary process in DD, by which indifference points would develop and differ as a function of delay length. If one boundary were found repeatedly, this would bias the walk toward this boundary. The origin bias function adjusted the origin of the walk along a sigmoidal function (Leibowitz, Baum, Enden, \& Karniel 2010) toward the previously found decision boundary via the formulas

if $v_{w}=v_{w-1},\left\{\begin{array}{c}o \rightarrow o+1 \\ o>40, o \rightarrow 40\end{array}\right.$

if $v_{w} \neq v_{w-1},\left\{\begin{array}{c}o \rightarrow o^{-1} \\ o<0, o \rightarrow 0\end{array}\right.$

where $v_{w}$ is the decision boundary found on walk $w$, and $o$ is a value that accumulates each consecutive time the boundary is found $(o=[0 \ldots 40])$ and is used to update the origin of the random walk via

originBias $=$ originScale $\left(\frac{1}{1+e^{-(0.5(o)-11)}}\right)$

where originScale sets the maximum the origin can be moved toward the decision boundary. The origin of the walk $\left(x_{1}\right)$ on the next walk $(w+1)$ was then set to the product of $\operatorname{originBias}(1 / v)$ if the upper decision boundary was found, or to $\operatorname{originBias}(-1 / v)$ for a lower-boundary decision. For example, if originScale $=.25$, then the random walk could be maximally initiated $25 \%$ closer to the decision boundary.

An additional term in the random walk was developed following initial simulations and also introduced in a subset of the experiments, to model how the prospective representation of the delayed choice, and its decay over time, might influence which decision boundary was found by the random walk. The "drift bias" decayed exponentially by modifying the distribution of the random walks $\mathrm{N}\left(d_{i}, 1\right)$, where

$d_{i}=\operatorname{scaleDriftBias}(-1 / v) * 0.95^{i}$, where $i=[0 \ldots n]$

and $n$, as previously, is the duration of the walk $(5,000$ steps for all simulations). The assumption of exponential memory decay is common to a number of models (Mensink \& Raaijmakers, 1988; Murdock, 1997).To quantify the fit of the model to the experimental data, the prediction error was calculated as

$P E=\sqrt{\sum\left(Y_{d}-\hat{Y}_{d}\right)^{2}}$

where $Y$ is equal to the mean value of the immediate lever from the experimental data at delay $d$, and $\hat{Y}$ is the mean value of the immediate reward boundary recovered from the model at delay $d$. 


\section{Results}

\section{Delay discounting}

One Wistar rat did not learn to lever press, and one Wistar and one $\mathrm{P}$ rat did not meet our inclusion criterion for magnitude discrimination $(70 \%$ of the maximum reward value of $6=$ 4.2 pellets), and were therefore excluded. No rat population differences were observed in the numbers of completed trials at any delay [main effect of rat population, $F(1,27)=1.145, p=$ .2940; Delay $\times$ Rat Population interaction, $F(5,135)=0.5886$, $p=.7087$ ] (see Fig. 1A). A clear preference for the largermagnitude delay lever was observed at the 0-s delay in both rat populations, thus indicating that each rat population discriminated the magnitude of the reward (Fig. 1B). Additionally, no rat population differences in indifference point were observed at the 0-s delay, indicating that the larger, delayed reward was valued similarly (Fig. 1C). A clear shift in the preferences for the delay lever was observed across delays [Choice Type $\times$ Delay interactions: P rats, $F(5,130)=151.2, p<.0001$;
Wistar rats, $F(5,140)=72.97, p<.0001]$, indicating that the animals understood the relationship between reinforcer size and the delay (Fig. 1B). The pattern of forced choice trials inversely mirrored that of choice trials in the expected direction - there were a greater number of forced choices to the delay lever across delays in both groups (due to discounting/choosing more consecutive immediate rewards), with $\mathrm{P}$ rats displaying more forced choices to the delay lever than did Wistar rats over delays (data not shown). Choice responding reached a stable asymptote by the final choice trial, indicating that animals titrated their choices on the basis of the delay within a trial.

The results of analysis of DD across delays can be seen in Fig. 1C. We observed significant main effects of delay $[F(5$, $135)=97.60, p<.0001]$, rat population $[F(1,27)=4.284, p<$ $.05]$, and a Delay $\times$ Rat Population interaction $[F(5,135)=4.21$ $p<.01]$. Post-hoc testing indicated that $\mathrm{P}$ and Wistar rats differed significantly at the 4 -s $(p<.05), 8$-s $(p<.01)$, and 16-s $(p<$ $.01)$ delays, with $\mathrm{P}$ rats displaying lower indifference points. Analyses of the areas under the curve derived from (1) the indifference points at each delay $[t(27)=3.46, p<.01$; see Fig. $1 \mathrm{C}$
A

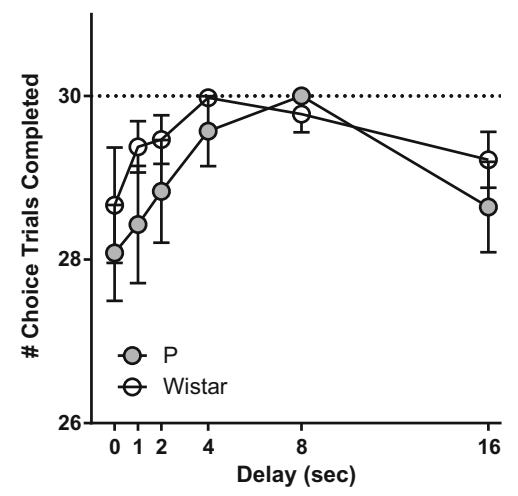

B

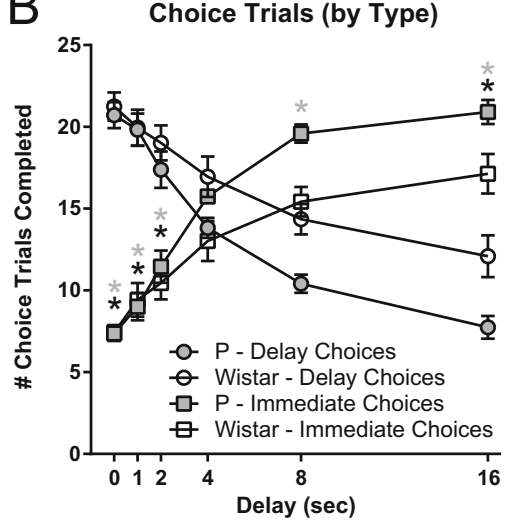

C

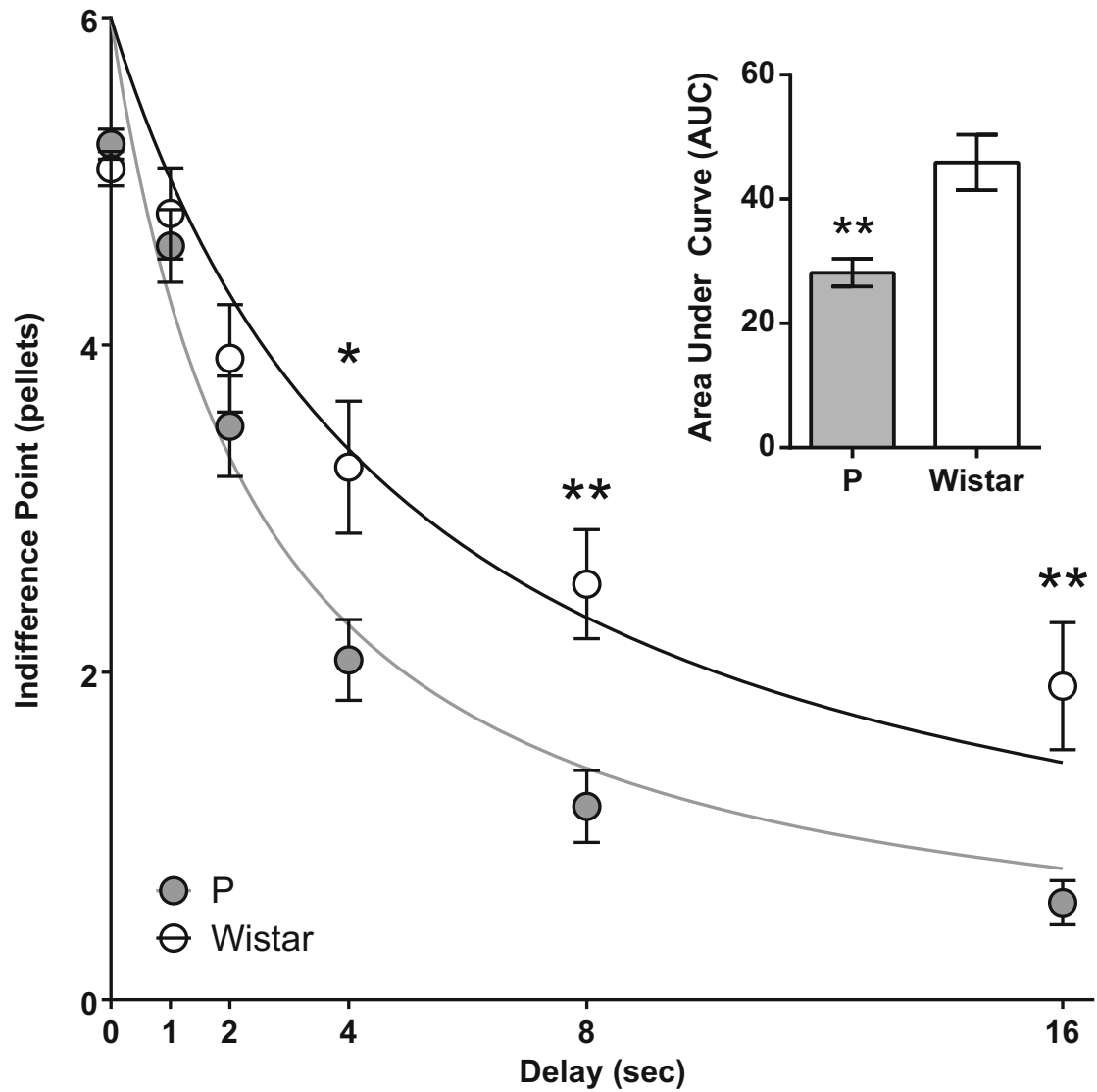

Fig. 1 (A) Slower delay discounting in Wistar versus $P$ rats. P and Wistar rats completed the same numbers of choice trials across delays. $(\mathrm{B}, \mathrm{C})$ However, a greater number of immediate versus delay choices were made in $\mathrm{P}$ rats over ascending delays (B), resulting in steeper discounting in $\mathrm{P}$ than in Wistar rats $(\mathrm{C})$. The asterisks in panel $\mathrm{B}$ indicate within-rat population differences in the mean numbers of immediate versus delay choices (gray $*=\mathrm{P}$ rat differences; black $*=$ Wistar rat differences). The asterisks in panel $\mathrm{C}$ indicate differences between the rat populations: ${ }^{*} p<$ $.05 ;{ }^{* * *} p<.01$ 
inset] and (2) the best-fit lines from nonlinear hyperbolic fitting $[t(27)=2.91, p<.01]$ both also indicated significant group differences, with lower values in $\mathrm{P}$ rats. To evaluate differences in the rates of discounting (i.e., the steepness of the best-fit hyperbolic curve), $k$ values were evaluated. The data were not normally distributed (Shapiro-Wilk: $W=0.83, p<.01$ ); thus, nonparametric Mann-Whitney tests were performed, which indicated significant differences between $\mathrm{P}$ and Wistar rats $[U(27)$ $=50, p<.05]$. The $k$ values were then natural-log-transformed to allow for analysis with parametric statistics (and comparison with other normally distributed variables). The results of this analysis supported the nonparametric results of rat population differences in $k[t(27)=2.76, p=.01]$, with steeper slopes in $\mathrm{P}$ rats. Thus, the rat populations differed in DD, with $\mathrm{P}$ rats displaying increased impulsivity as compared to the Wistar rats.

\section{Lever consistency}

To further explore the cognitive processes that underlie DD, the extent to which the same lever was pressed to initiate a trial and subsequently make a choice response was quantified (i.e., initiation/choice "consistency"). This was based on the premise according to which our study was designed: that consistent trials would reflect choice decision intent (i.e., a prospective decision strategy). The probability that trials were consistent depended on the frequency of delay- versus immediate-lever initiations, as well as the frequency of delay- versus immediate-lever choices. The chance probability of a consistent trial for each animal/ subject $(s)$ at each delay $(d)$ was calculated as

$$
\begin{aligned}
p\left(\text { consistent choice }_{n, d}\right)= & \left(\frac{D_{i, s, d}}{T_{i, s, d}} * \frac{D_{c, s, d}}{T_{c, s, d}}\right) \\
& +\left(\frac{I_{i, s, d}}{T_{i, s, d}} * \frac{I_{c, s, d}}{T_{c, s, d}}\right),
\end{aligned}
$$

A Initiation/Choice Consistency

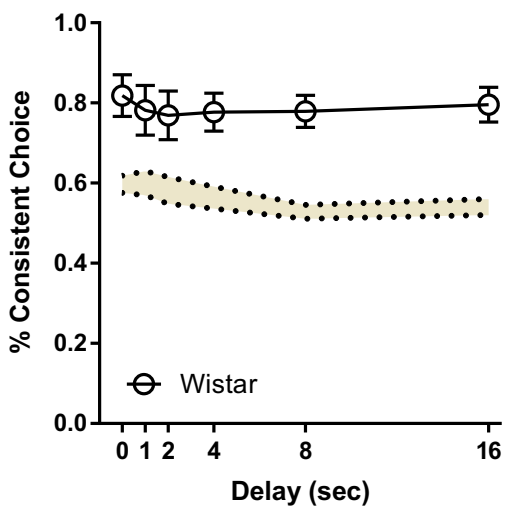

Fig. 2 Slower delay discounting in Wistar rats is paralleled by consistent responding across the initiation and choice epochs. Wistar rats (A) displayed greater-than-chance initiation/choice consistency across delays, where $D$ and $I$ represent responding on the delayed or the immediate lever, respectively, and $T$ is the total number of choices. The subscripts $i$ and $c$ represent choices during the initiation or choice phase, respectively. Thus, each animal had its own chance-level probability of making consistent responses, which was derived from the observed pattern of initiations and choices. An analysis of the chance probabilities (shaded lines in Fig. 2A, B) indicated no differences between the rat populations. However, we did observe a significant main effect of delay $[F(5,135)=6.57, p<.0001]$, with the chance probability of consistent choices decreasing with increasing delay length. Analysis of the observed consistency revealed a significant main effect of delay $[F(5,135)=4.10, p<.01]$ driven almost entirely by $\mathrm{P}$ rats, a main effect of rat population $[F(1,27)=$ $15.26, p<.001]$, and a significant Delay $\times$ Rat Population interaction $[F(5,135)=2.64, p<.05]$. Bonferroni-corrected post-hoc testing detected differences between the observed consistencies between rat populations at the 4-s $(p<.01), 8$-s $(p<$ $.001)$, and 16-s $(p<.001)$ delays, with Wistar rats displaying higher consistency than $P$ rats at each of these delays. An analysis of the chance versus the observed consistency revealed a main effect in Wistar rats $[F(1,28)=21.04, p<.0001]$, driven by higher consistency than chance at all delays $(p s<.05$; Fig. 2A). In P rats, there were no differences in the chance probability versus the observed consistency $(p s>.30$; Fig. 2B). Thus, Wistar rats displayed levels of consistency greater than would be expected by chance, whereas Ps did not. This indicates that a behavior plan guiding the choice at the initiation phase of the task was maintained through the choice phase in Wistars.

\section{Response latencies}

Separate analyses were conducted on latencies to make choices of each of the two possible levers. In these analyses,

\section{B Initiation/Choice Consistency}

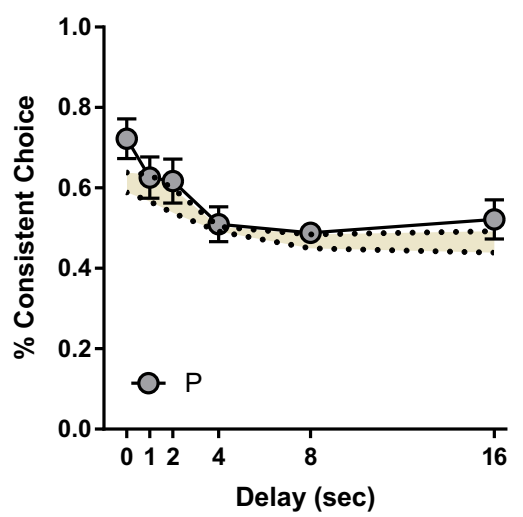

whereas $\mathrm{P}$ rats (B) did not. The yellow shaded regions denote the \pm SEMs of the expected probabilities of a consistent choice at each delay 
the median response time latency was taken for each animal over the last three days at each delay, and the mean of this value was used for the statistics. For immediate-choice latencies, there was a significant main effect of delay only $[F(5$, $135)=10.37, p<.0001 ;$ Fig. $3 \mathrm{~B}]$, with generally longer latencies at the 1-s and 2-s delays than at the other delays. However, it is important to note that the latencies at the 1-s and 2-s delays were not significantly greater than those at the other delays. For delay-choice latencies, we found a significant Delay $\times$ Rat Population interaction $[F(5,135)=5.43, p=$ .0001 ; Fig. $3 \mathrm{C}]$, which post-hoc testing indicated was driven by significantly slower responding in $\mathrm{P}$ rats at the 8 -s and 16 -s delays $(p s<.05)$.

\section{Relationships between behavioral measures}

To evaluate the relationship between the measures of impulsivity, lever consistency, and latency to make choices, we performed correlation and linear regression analyses. Initiation/ choice consistency was significantly negatively associated with
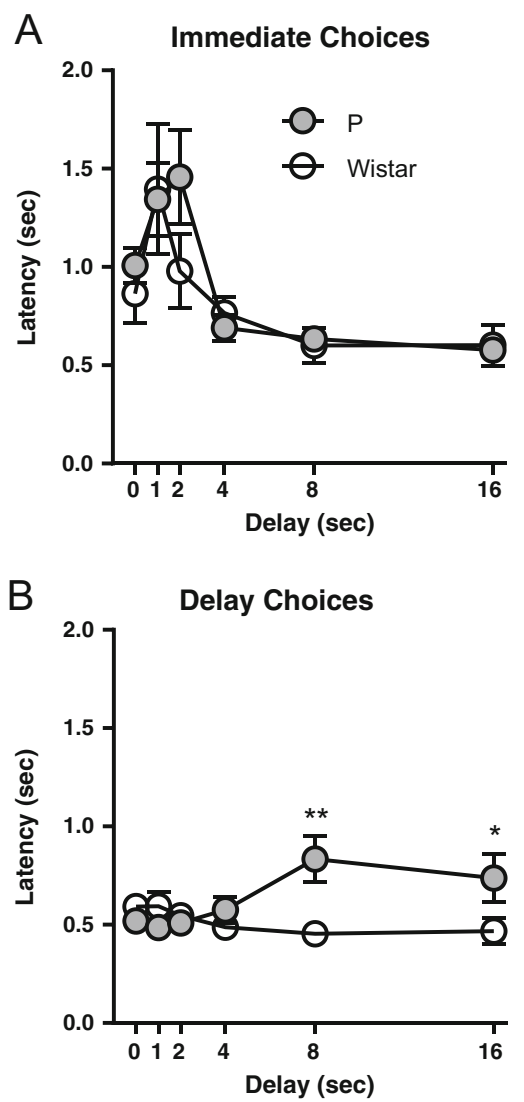

Fig. 3 Increased decision times are observed in P rats at long delays. (A) When stratified by choice types, no differences were observed between Wistar and $\mathrm{P}$ rats in latencies to make immediate choices. (B) However, delay-choice latencies in Ps at the 8-s and 16-s delays were longer, relative both to earlier delays and to Wistars at the same delays. ${ }^{*} p<.05 .{ }^{* * *} p<$ .01 the natural-log-transformed $k$ values (i.e., impulsivity) in Wistar rats $(r=-.57, p<.05)$, but not in $\mathrm{P}$ rats $(p=.24$; Fig. 4A). Initiation/choice consistency was also significantly negatively associated with choice latencies in Wistar rats $(r=$ $-.54, p<.05)$, but not in $\mathrm{P}$ rats $(p=.70$; Fig. 4B). However, $k$ values were significantly positively associated with choice latencies in P rats $(r=.66, p<.05)$, but not in Wistar rats $(p=.17$; Fig. 4C). Thus, for Wistar rats, the more consistent the less impulsive and the faster the choice decision, and for $\mathrm{P}$ rats, the slower the choice, the more impulsive.

\section{Drift diffusion model}

The model was parameterized (see below) so that its values could be interpreted similarly to those from the experimental data. For example, the decision boundaries were set at $1 / v$ so that the immediate-decision boundary could be roughly interpreted as the number of pellets. In addition, the diffusion constant was fit so that one step corresponded to $\sim 10 \mathrm{~ms}$ in the experimental data (the sampling rate of the behavioral hardware), resulting in similar response time distributions [twosample Kolmogorov-Smirnov test: $D(100)=0.0875, p \geq .05$ ] between the experimental data (Fig. $5 \mathrm{C} 1$ ) and the model (Fig. 5C2).

The experimental data were acquired via the within-session adjusting-amount DD procedure. This DD procedure is attractive due to the fact that it allows us to calculate an indifference point that provides an accurate within-session measurement of the subjective value of the delayed reward. In both the experimental data and the model, the value of the immediate lever was dynamic on early walks/trials, and it eventually reached asymptote based on either the distance of the delayed boundary from the origin (Fig. 5D2) or the duration of the delay (Fig. 5D1); thus, the model (even prior to addition of bias features) re-created this important feature of the DD procedure.

The value of the actual reward $v$ could be manipulated by changing the discounting function $k$. Manipulating $v$ to reflect changes in the value of the reinforcer created a hyperbolic discounting function, in which the probability of finding the delayed boundary decreased as a function of delay. To explore whether simply modifying $k$ was sufficient to re-create the observed discounting functions from the experimental data, the experimentally derived $k$ values $\left(k_{e}\right)$ were initially used in the simulations. Upon providing the model with the $k_{e}$ values observed in the experimental data $\left(k_{\langle e, w\rangle}=0.1963\right.$ to simulate the Wistars, and $k_{\langle e, p\rangle}=0.4054$ to simulate the P rats), clear discounting was observed in each condition, and discounting was slowed in simulated Wistars relative to simulated Ps (Fig. 5E). However, the $k$ values recovered from these simulations $\left(k_{m}\right)$ were much higher than those observed in the experimental data. In the case of the Wistars, the experimental $k_{\langle e, w\rangle}=0.1963$ was -5.1 standard deviations lower than those observed in the simulations $\left(k_{m, w}=0.3327 \pm 0.027\right.$, one- 
A Consistency vs. $k$

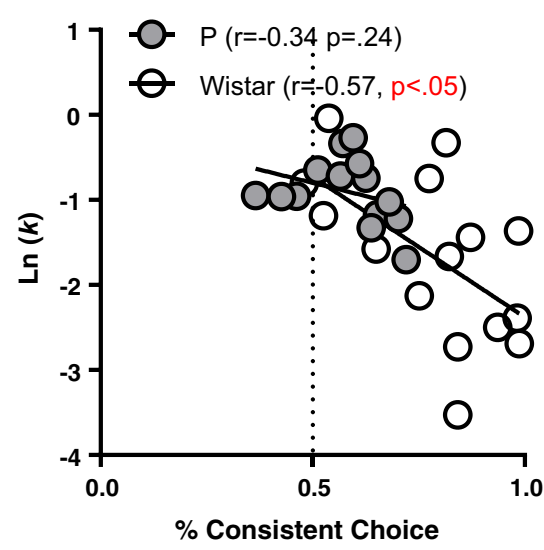

B Consistency vs. Latency

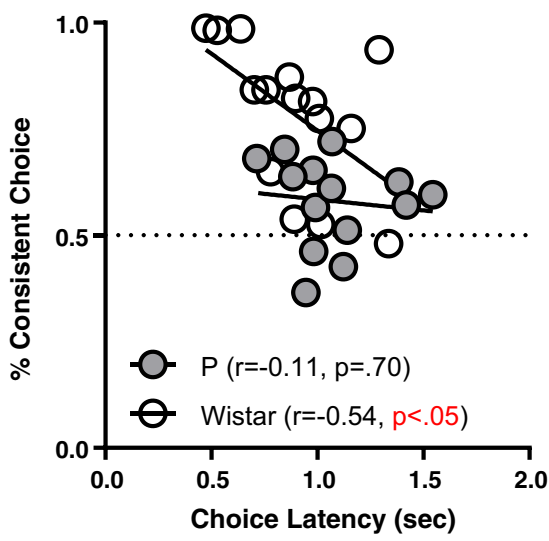

C

Latency vs. $k$

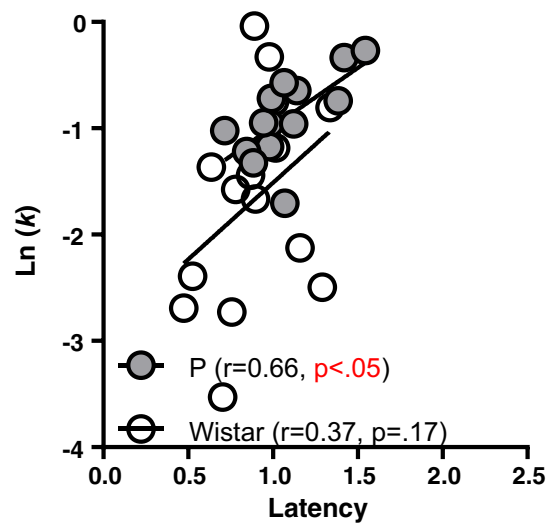

Fig. 4 Impulsivity is correlated with choice consistency and latency. (A) Choice consistency was significantly negatively associated with $k$ in Wistar rats only: the more consistent, the less impulsive. (B) Choice consistency was significantly negatively associated with choice latency in Wistar rats only: the more consistent, the faster the choice responses. (C) Choice latency was significantly positively associated with $k$ in $\mathrm{P}$ rats only: the more impulsive, the slower the choice responses

sample $z$ test, $p<.0001)$. In simulated P rats, the experimental $k_{e, p}=0.4054$ was -3.08 standard deviations lower than those observed in the simulations $\left(k_{m, w}=0.5219 \pm 0.038\right.$, one- sample $z$ test, $p=.0021$ ) (Fig. 5E, inset). Thus, by only changing $k$, the discounting functions created by the model did not adequately re-create the discounting observed in either population, suggesting that additional parameters should be considered to better describe discounting in each rat population. Furthermore, the discounting parameter, $k$, in its pure form, provides only a mathematical description of how the perception of the time interval influences the perceived reward magnitude, which could each be influenced by a number of $\operatorname{cog}$ nitive processes.

A number of observations in the behavioral data shed light on the differences in decision making that led to altered DD between the rat populations. First, Wistars discounted more slowly (Fig. 1C), and slower discounting was correlated with a propensity to respond on the to-be-selected choice lever at initiation (Fig. 4A). These data suggest that, in Wistars, a relationship exists in the lever selected to initiate a trial and the lever selected as the choice. There were no differences between $\mathrm{P}$ and Wistar rats in the numbers of initiation decisions on either the delay or the immediate lever [main effect of rat population: $F(1,27)=0.13, p \geq .7$; Rat Population $\times$ Delay interaction: $F(5,135)=0.92, p \geq .4$, but there was a slight bias in favor of initiating trials on the delay lever in both groups ( $\approx 66 \%$ delay initiations in Ps, vs. $\approx 64 \%$ delay initiations in Wistars). Since P and Wistar rats tended to initiate a trial on the delay lever with equal probabilities, this rules out an initiation bias as the cause for population differences in discounting. Rather, this suggests that, in Wistars, the initiation choice reflects a prospective strategy that may guide the decision about which lever is chosen during the choice phase. This is supported by the observation that the Wistars that were the most consistent also exhibited faster response latencies (Fig. 4B). In addition, at the 8-s and 16-s delays, Wistars were faster to choose the delay lever than were $P$ rats, whereas there were no differences between the populations for immediate choices (Fig. 3C). Consistency between the initiation and the delay lever may reflect a process through which a bias for the preferred lever accumulates over trials, reducing the need for deliberation as the preferred choice is more clear. Alternatively, the consistent choices may reflect a prospective process whereby the animal forms and maintains a trace of the to-be-selected lever, which then guides the decision (i.e., facilitates the mapping of the delayed reward to the context). These possibilities were each modeled separately as additional parameters, to explore potential differences in discounting between the $\mathrm{P}$ and Wistar rats. For all subsequent simulations, the $k_{e}$ value used in the model was 0.3009 (the mean of the Wistar and P rats; Fig. 5E, black circles in main figure).

To explore how an accumulating bias based on past choices might affect decision making, the origin of the random walk was updated on the basis of which boundary had been found on the previous trial. This could reflect a number of cognitive constructs, such as value judgments about past choices (was it 
A
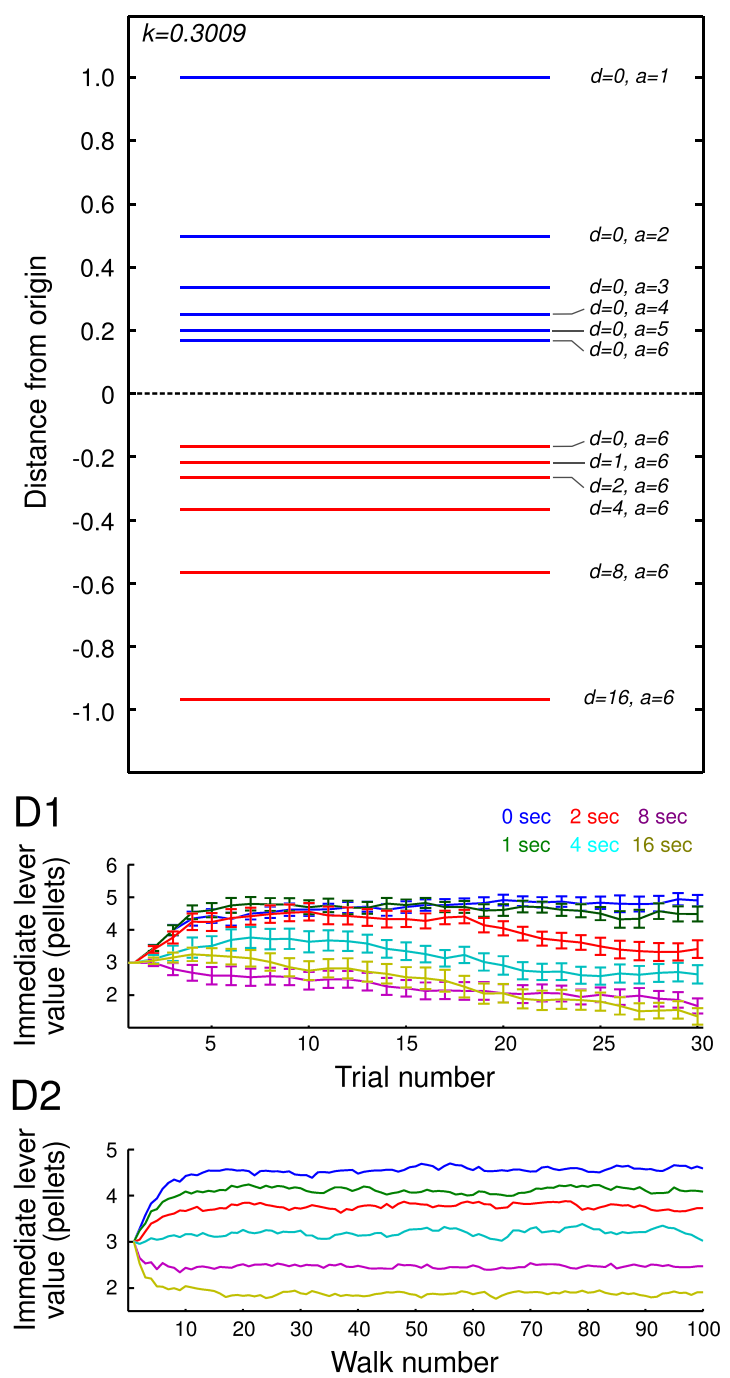

B

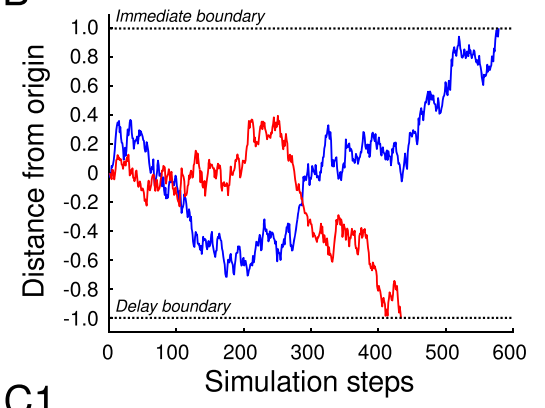

C1

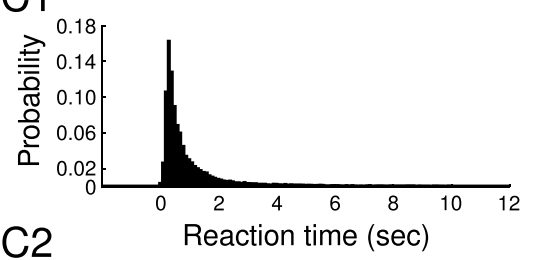

C2
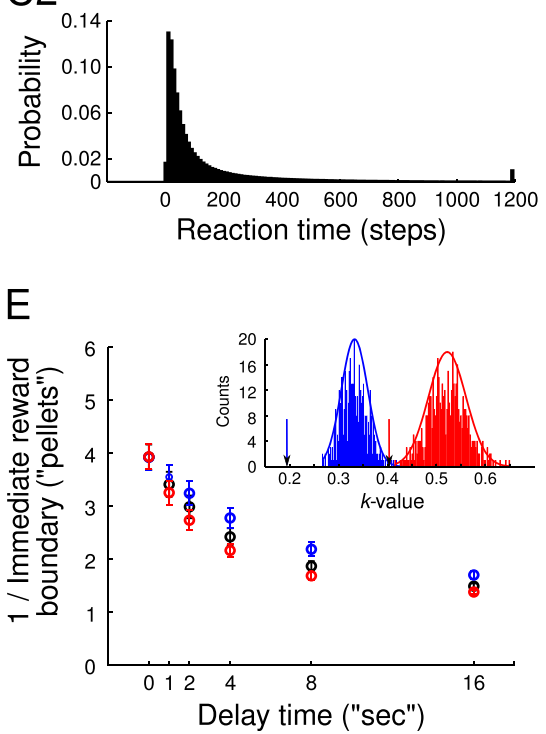

Fig. 5 Description and performance of our drift diffusion model of delayed discounting. (A) A figure describing how each decision boundary is altered by changing the delay $(d)$ and the value of the reinforcer $(a)$, with different colored lines to denote the delay (lower) boundaries (red in the online version) and immediate (upper) boundaries (blue online). (B) Representative random walks are shown that reach the delay and immediate boundaries (dashed lines). (C) Response time distributions for all trials acquired in the experimental data $(\mathrm{C} 1)$ and all walks in the model data $(\mathrm{C} 2)$. Notice the similarity in the shapes of the distributions. (D1) Values (means \pm SEMs) of the immediate lever at each trial in the experimental data [Trial $\times$ Delay interaction: $F(115,3450)=6.84, p<.0001]$. (D2) Mean values of the

worth waiting for the delayed reward?), integrating the temporal information about the delay (how long was the delay?), building an association between the temporal delay and the number of pellets available immediately, or the decision process becoming increasingly easier. As such, the accumulation of information about past choices, and how they might relate to the current choice, was modeled as a sigmoidal functionthe origin bias (see the originBias equation). Modifying scaleOriginBias alone had a modest effect on the $k$ values immediate boundary for the model data. The colors of the lines in panels D1 and D2 correspond to the same delays. Notice that each line eventually reaches a unique asymptote (i.e., indifference point), depending on the delay. (E) Simulation results using the $k$ values from the experiments in each rat population. The discounting functions (means $\pm S D$ s) are plotted for simulated Wistars (blue) and $\mathrm{P}$ rats (red), and for the mean $k$ values from Ps and Wistars (black). The inset in panel E shows the distribution of $k$ values generated by the model for 500 bootstraps (with different color bars indicating simulated Wistars and Ps). The arrows denote the experimental $k$ values for each rat population; notice that they are much lower than the simulated values

recovered from the simulations (Fig. 6A). Although it was possible to recover $k$ values from the model similar to those observed in the $\mathrm{P}$ rats by modifying scaleOriginBias alone, modifying this variable did not yield $k$ values similar to those observed in the Wistar rats. Modifying scaleOriginBias resulted in discounting functions with an increased probability that the random walk would reach the delay boundary at short delays $(0,1 \mathrm{~s})$ and the immediate boundary at long delays $(8$ and $16 \mathrm{~s}$; Fig. 6A2). 
Prospective memories are conceptualized as transient representations of future actions (Crystal \& Wilson, 2015). To explore whether a prospective process could account for alterations in discounting, random walks were transiently biased toward the delay boundary by using the scaleDriftBias term. The walk was biased toward the delay, and not the immediate, boundary for the following reasons: First, there is evidence that the ability to withhold an impulsive response requires that a representation of the delayed reward must be formed and maintained (Buckner \& Carroll, 2007; Kurth-Nelson et al., 2012). Second, each rodent population exhibited an initiation bias for the delay lever, indicating the higher incentive motivational value of this lever. Additionally, an immediate decision does not likely require the level of planning and maintenance of information about the to-be-made as compared with the delay choice. This was supported by the observation that the Wistars that chose the delay lever most often (e.g., the slowest discounters) were the ones that were the most consistent (Fig. 4A). Further support for this idea was observed as an increase in the latency to choose on immediate versus delay choices [main effect of choice type: $F(1,28)=8.57, p=.0067$ ] and a strong relationship between choice latency and consistency in Wistars (Fig. 4B).

We hypothesized that if a memory of the incentive motivational value of this lever can be maintained up to the choice, then this can bias the choice process. Indeed, the inclusion of the scaleDriftBias term dramatically slowed discounting and was capable of yielding $k_{\langle m\rangle}$ values in the range observed in the experimental data for both Wistar and P rats (Fig. 6B1). An example in which scaleDriftBias $=0.20$, and scaleOriginBias $=0$ is shown in Fig. 6B3. After including this variable, the $k_{\langle m\rangle}$ values recovered from the simulations were $0.27335 \pm 0.024$ ( $\mu \pm \sigma$ ), which slowed discounting relative to when scaleDriftBias $=0\left(k_{\langle m\rangle}=0.4294 \pm 0.035\right)$.

Although the $k_{\langle e, p\rangle}$ values of the P-rat experimental data could be re-created by changing the scaleOriginBias or the scaleDriftBias parameter (gray circles in Fig. 7A1), the fit of the model to the P-rat experimental data was best (e.g.,
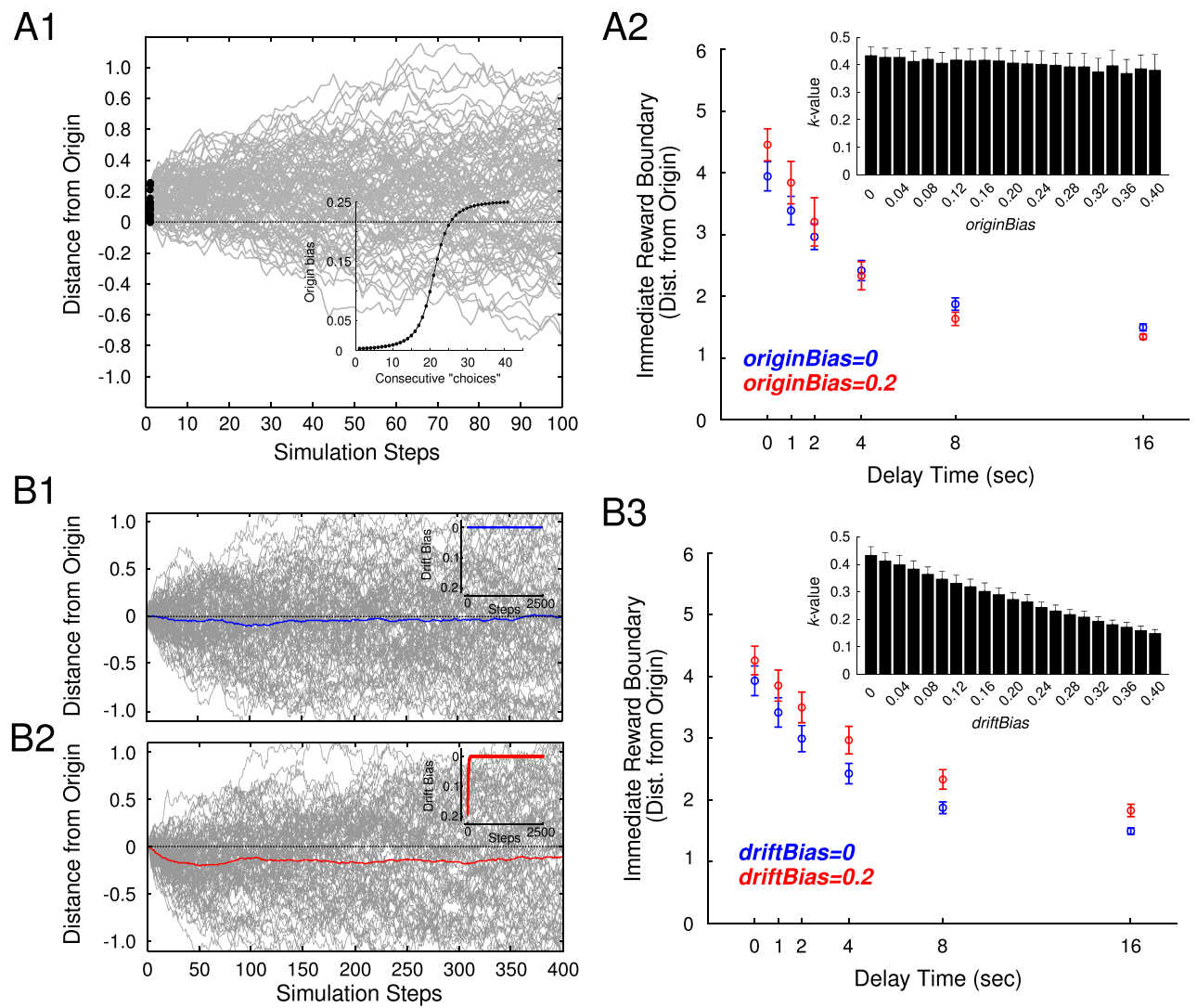

Fig. 6 Performance of the origin and drift biases in simulations of delay discounting. (A) Performance of the origin bias. (A1) Representative walks for a 16 -s delay with origin bias $=0.2$. Notice how the origin of the random walk moves closer to the immediate boundary (top). The inset shows the origin bias function, in which the origin is incrementally moved toward a decision boundary on the basis of how often it was found on previous choices. (A2) The resulting discounting functions for an origin bias $=0$ or 0.2 (different color symbols). The inset shows how $k$

values are altered by increasing the origin bias. (B) Performance of the drift bias. Random walks are shown in which the driftBias $=0$ or 0.2 , in panels B1 and B2, respectively. Here, the different color lines are the means of all random walks, and the insets show the driftBias at each step of the walk. In panel B3, the discounting functions are shown for driftBias $=0$ or 0.2 (different color symbols). The inset in B3 shows how $k$ values are altered by increasing the drift bias. All of the data in panels A2 and B3, including the insets, are presented as means $\pm S D \mathrm{~s}$ 
prediction error was minimized) when the scaleOriginBias $=$ 0.36 and scaleDriftBias $=0$ (Fig. 7A3), indicating that changing the origin bias more adequately modeled the P-rat experimental data.

A number of combinations of the origin and drift bias existed that yielded $k_{\langle m\rangle}$ values close to those observed in the Wistar experimental data (i.e., several parameter combinations generated low prediction errors; see the black circles in Fig. 7A1, A2). However, the parameters that best minimized the prediction error (Fig. 7) were scaleOriginBias $=0.2$ and scaleDriftBias $=0.26$. The improvement in the fit of the model to the Wistar data was small when scaleOriginBias $=0$, indicating that although this parameter made improvements in the fit, the drift bias was far more important in creating an optimal fit to the experimental data in Wistar rats.

If Wistar rats formed a plan of their choice at the initiation of the task and maintained this in memory, one would expect a faster response time at the choice. In the experimental data, faster response latencies for delayed rewards were observed in Wistar rats at 8-s and 16-s delays (Fig. 3C). To explore this finding in the model, we used the parameters for the origin and drift bias that optimally re-created the $k_{\langle m\rangle}$ values and minimized prediction errors for each rat population (see above). As in the experimental data, a Rat Population $\times$ Choice (immediate vs. delay) interaction $[F(1,499987)=76,614.43, p<$ $.0001]$ was observed for the natural-log-transformed response times recovered from the model. In addition, the distributions of the response times for an immediate versus a delay response were more similar in the modeled Wistars (Kullback-Leibler divergence $=1.89$ ) than in the modeled Ps (Kullback-Leibler divergence $=5.53$; Fig. $7 \mathrm{C} 1, \mathrm{C} 2)$. Collectively, these data indicate that, as in the experimental data, optimizing the model parameters on the basis of the Wistar data decreased the latency of the random walk to find the delay boundary. Thus, the inclusion of the origin bias optimized the fit of the model to the P-rat experimental data, and the Wistar experimental data
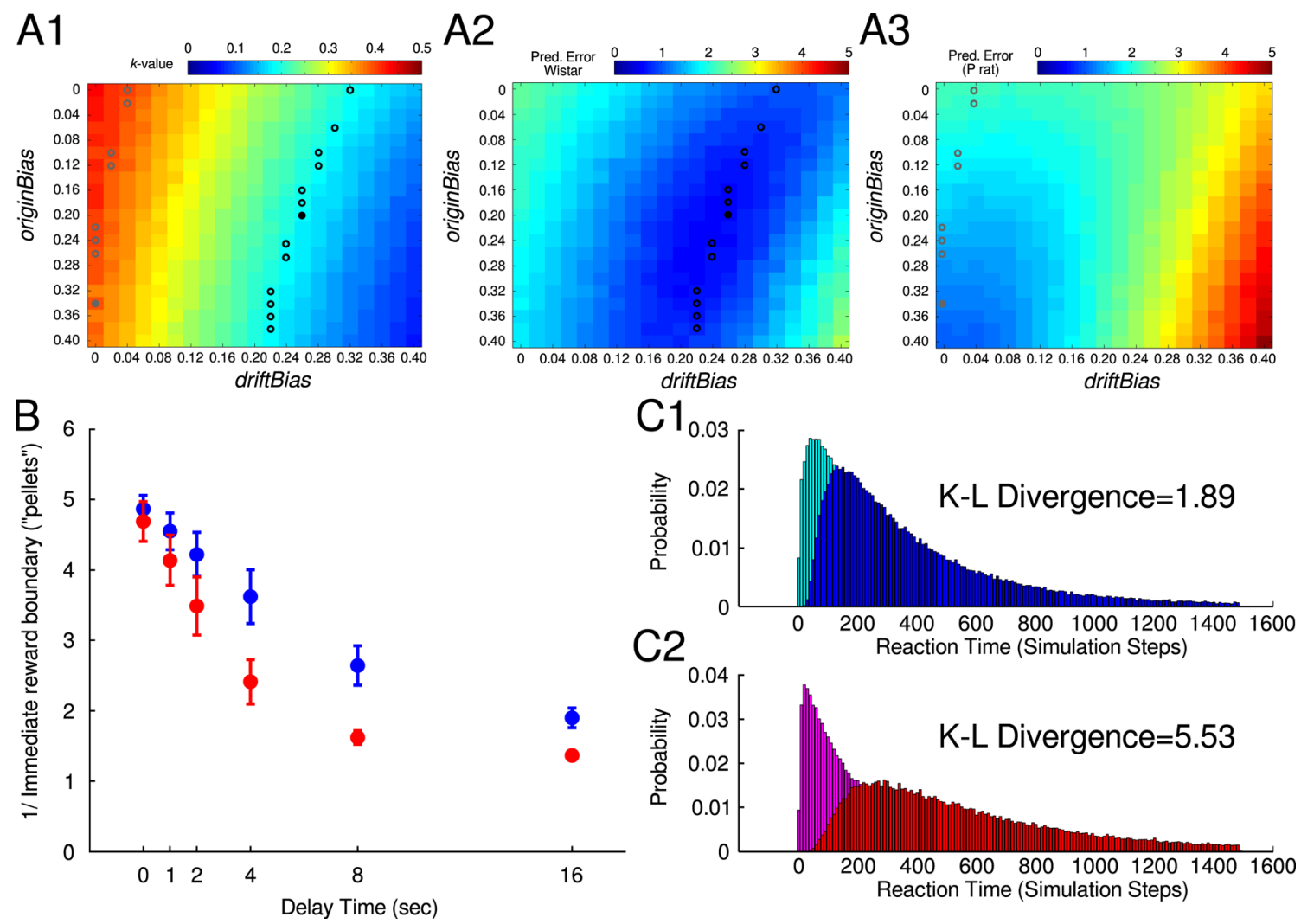

Fig. 7 Optimal model parameters to fit the Wistar and $\mathrm{P}$ rat experimental data. A1 Mean $k$ values recovered from simulations (100 bootstraps) of delay discounting after altering the origin and drift biases. The open circles on the heat plots indicate where the mean $k$ values of the simulations were within \pm 0.01 of the experimental data, and the two closed circles denote the origin and drift bias combinations that yielded the $k$ values that were closest for the rat populations (gray $=\mathrm{P}$ rats, black $=$ Wistars). Model fits were further evaluated by measuring the prediction errors of the mean $i$ values at each delay in the model and the experimental data, for both Wistars (A2) and P rats (A3). The overlaid

circles are identical to those in panel A1. (B) The resulting discounting functions for the origin and drift bias parameters that optimally fit the Wistar (blue online) and $\mathrm{P}$ rat (red online) experimental data. C Average Kullback-Leibler (K-L) divergences in Wistar (C1) and P (C2) rats between the simulated response time distributions of immediate (cyan/magenta online) versus delay (blue/red online) choices. There was a greater difference in response times between immediate and delay choices in P rats $(\mathrm{C} 2)$, with longer latencies on delay choices than on immediate choices. All data are presented as means $\pm S D \mathrm{~s}$ 
were optimally fit by including the drift bias. Taken together, these data highlight potential differences in the strategies employed by each rat population to guide decision-making, and further suggest that the ability to effectively plan a decision is key to mitigating an impulsive choice.

\section{Interlever response interval}

We next designed a set of experiments to evaluate the influence of inserting a delay between the initiation lever and access to the choice lever in the subset of Wistar rats. The primary goal of these experiments was to determine whether increasing the temporal delay between trial initiation and choice availability would interfere with lever consistency. We hypothesized that increasing the IRI delay would interfere with consistency and increase the choice latency.

At all three reward delays, we found significant IRI $\times$ Consistency interactions $[0 \mathrm{~s}: F(4,64)=7.02, p \leq .0001$; 4 s: $F(4,64)=3.95, p \leq .01 ; 16$ s: $F(4,64)=3.95, p \leq .01]$. Post-hoc testing indicated above-chance differences in consistency at the 1 -s $(p s \leq .001)$ and 2 -s $(p s \leq .001)$ IRIs at all three reward delays, and above-chance differences at the 4-s ( $p s \leq$ $.01)$ and 8 -s $(p s \leq .05)$ IRIs at the 4-s and 16-s reward delays (Fig. 8). At all three reward delays, the 16-s IRI decreased consistency to chance levels. Thus, longer IRI intervals decreased consistent lever responding.

IRI length increased choice latencies at all reward delays (main effect of IRI). Interestingly, significant increases in choice latencies were only observed at IRI lengths at which consistency was no greater than chance. These results support the observation that the consistent lever-press strategy in Wistar rats leads to decreases in latencies to choose a reward. IRI had no impact on indifference points as a function of IRI delay (no main effect or interactions of IRI).

\section{Discussion}

These results support recent work demonstrating that alcoholpreferring "P" rats display increased impulsivity relative to other alcohol-preferring, non-alcohol-preferring, and heterogeneous rat strains (Beckwith \& Czachowski, 2014; Perkel et al., 2015), as well as reports of greater impulsivity in other populations of selectively bred alcohol-preferring rodents (Mitchell, 2011; Oberlin \& Grahame, 2009; Wilhelm \& Mitchell, 2008). These data are also consistent with human studies that have shown a relationship between impulsivity, measured via DD, and those with a family history $(\mathrm{FH}+)$ of an AUD (Mitchell, 2011). Although these data clearly indicate a relationship between impulsivity and family history of excessive drinking, the relationship between impulsivity and family history does not appear to extend to all $\mathrm{FH}+$ participants or rodents selectively bred for excessive drinking (Beckwith \&
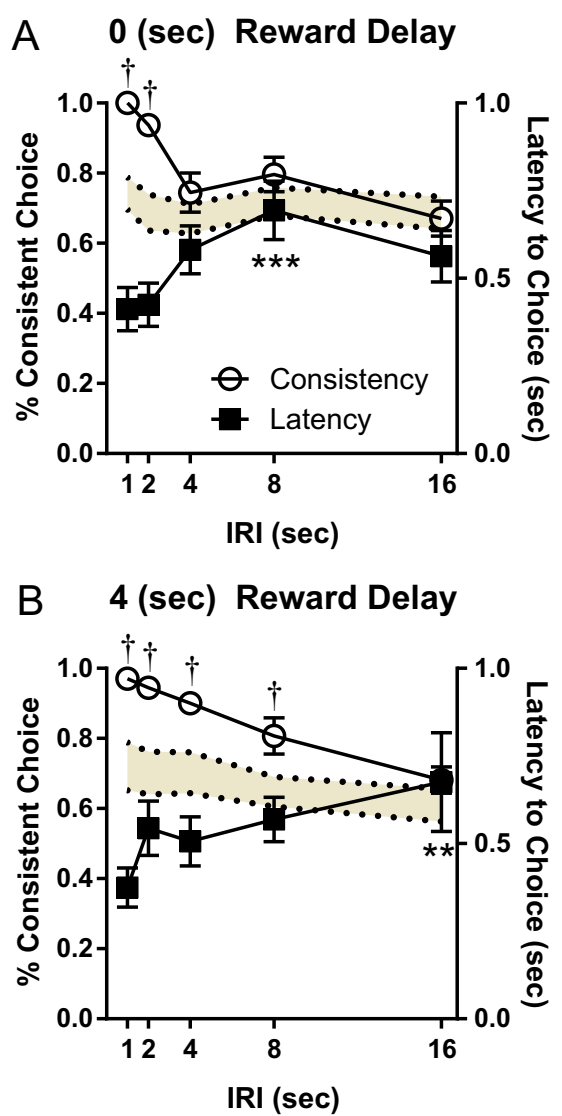

C 16 (sec) Reward Delay

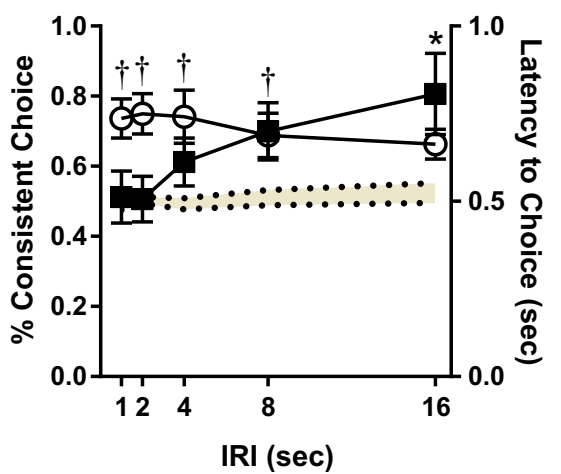

Fig. 8 Influences of interlever response interval (IRI) on consistency and on choice latencies in Wistar rats. As consistency decreased to chance levels across IRIs, choice latency increased - an effect that was observed at all three reward delays tested: (A) 0 -s reward delay. (B) 4-s reward delay. (C) 16-s reward delay. ${ }^{\dagger}$ Differences in consistency from chance ( $p$ s $<.05$ ). Asterisks indicate differences from the 1-s IRI with a given reward delay: ${ }^{*} p<.05 ;{ }^{* *} p<.01 ;{ }^{* * * *} p<.001$

Czachowski, 2014; Wilhelm \& Mitchell, 2012; Wilhelm, Reeves, Phillips, \& Mitchell, 2007). These studies highlight the need to further refine our understanding of the various cognitive functions that influence impulsivity and the specific modalities of alcohol drinking each is related to.

In the experimental data used to fit the model a clear preference for the delay lever was observed at the 0-s delay 
(Fig. 1B), increasing delays also reduced the probability of choosing the delay lever (Fig. 1B), and a stable choice responding was observed at the end of the trial (Fig. 5D1). Collectively these data indicate that both $\mathrm{P}$ and Wistar rats demonstrated mastery of the behavioral task. A previous study, also employing a computational model, found no evidence that adjusting the delay influenced decision-making in a withinsession adjusting delay task (Cardinal, Daw, Robbins, \& Everitt, 2002). However, a number of experimental and procedural differences prevent the comparison of these data to the model presented herein.

It is possible that the light cue remaining on following choice decisions exacerbated the difference in DD between the two different rat populations given that cues marking delay choices have been shown to increase DD in groups of highly impulsive animals and decrease DD in groups of animals with low impulsivity (Zeeb, Floresco, \& Winstanley, 2010). That this cue was present regardless of which reward lever was chosen in our studies versus only the delay choice in the study referenced above, and given the fact that it remained on up to many seconds after reward delivery until trial end (24-s choice latency) makes this less likely in our view, but still a possibility.

In the present study, a clear relationship was observed in the behavioral strategy used (e.g., consistency) and DD - but only in Wistar rats (Figs. 1, 2, and 4). No detectable strategy was observed in $\mathrm{P}$ rats (Figs. 2B and 4A), who discounted steeply (Fig. 1). The effect of an animal's "family history" on consistency was robust and reproducible across all reward delay conditions tested (cf. Fig. 2A, B). This observation was key to better understand why P rats discounted more steeply than Wistar rats, and to further explore this observation, a drift diffusion model of DD was employed. Drift diffusion models are attractive because by modifying a few, well-defined parameters, a number of phenomena related to decision-making can be modeled.

As in previous studies, a random walk was used to model the cognitive search process (Kurth-Nelson et al., 2012), whereby information about the reward and delay are integrated to guide decision-making. The origin of the walk and the mean of the distribution from which the random walk was constructed, were each initially set to zero, which lead to a search process that was only influenced by reward magnitude and delay (i.e., the location of the decision boundaries). The decision boundaries, however, reflect an environmental variable that is external to the cognitive search process. In this regard, the cognitive search process was initiated de novo on each walk. However, as the animal progressed through the task, it was likely that information acquired from previous trials influenced the search process. In line with this view, it has been shown that information gleaned from previous trials, such as value judgments, can shape future decisions (Steiner \& Redish, 2014).

Sequential-sampling algorithms are attractive, given their ability to accurately model response time distributions (Brown
\& Heathcote, 2008; Ratcliff \& McKoon, 2008; Rodriguez, Turner, \& McClure, 2014). Response time distributions in two-choice decision-making procedures are typically positively skewed, and in the present study, this phenomenon was also observed in both the experimental and the modeled data (Figs. 3A and 5C). As choices become more difficult, or in situations in which accuracy is stressed over speed, response times increase (Bogacz, Wagenmakers, Forstmann, \& Nieuwenhuis, 2010). Temporally discounted rewards have also been shown to result in longer response times for choice selection (Beckwith \& Czachowski, 2014; Oberlin \& Grahame, 2009; Rodriguez et al., 2014; Roesch, Taylor, \& Schoenbaum, 2006). The geometry of the model presented herein leads to an increase in response times with increasing delays, which is consistent with previous experimental and modeling data.

Increases in response times with increasing delays may be related to the fact that as a reward becomes more temporally distant, it takes longer for the cognitive search process to map the reward to its context (Kurth-Nelson et al., 2012). However, a linear increase in response times across delays was not observed in our experimental data, suggesting that the search process was altered in our task as compared with those previously reported (Fig. 3). For delayed choices, response times tended to decrease in Wistar rats across delays, whereas they increased in P rats at the 8-s and 16-s delays (Fig. 3). The observed delay-choice response times suggest that the search process was facilitated somehow in Wistar rats and, at short delays, in P rats. For immediate choices, response times across delays were complex. In both rat populations, response times increased up to the 2-s delay, and then dropped dramatically at the 4-s to 16-s delays. This observation suggests that decision-making is a dynamic process that is influenced by genetic background, evolves across delays, and is possibly influenced by cognitive strategy.

When the experimentally derived $k_{\langle e\rangle}$ values were used in the model, the resulting fit of the discounting functions was poor in terms of the recovered $k_{\langle m\rangle}$ values and the mean immediate boundary values observed at each delay. The recovered $k_{\langle m\rangle}$ values from the model were considerably higher than those observed experimentally (Fig. 5E), and the function was "compressed" as compared to the experimental data (i.e., the immediate-lever values tended to be too small at short delays and too large at long delays). This suggested that parameters other than $k$ value should be considered, which motivated the decision to explore how biasing the random walk changed the fit of the model to the experimental data.

The origin bias was introduced to model a decision-making strategy in which the current choice was biased by information gleaned from the previous choices. In other words, finding one boundary on previous walks increased the probability of finding it in the future, which could reflect the decision process becoming easier (i.e., little deliberation was required). 
The inclusion of this parameter improved the fit of the $k_{m}$ values and minimized the prediction error of the model to the experimental data. In addition, this parameter increased the mean immediate boundary value at the 0 -s delay, indicating that the walk was increasingly biased toward the delayed boundary. Thus, the model data suggest that, when no delay was present, making the decision-making process easier was necessary to optimally model the experimental data. However, whereas the inclusion of the origin bias optimized the fit of the model to the $\mathrm{P}$ rat data, this was not sufficient to model the Wistar data.

The Wistar, but not $\mathrm{P}$ rats, consistently initiated a trial on the lever that was ultimately chosen in the choice epoch. This suggested that a behavioral plan was formed at, or close to, the initiation of the trial and was used to guide responding in the choice epoch. This was interpreted as Wistar rats forming a prospective memory of their choice, thus reflecting a "proactive" mechanism of behavioral control (Braver, 2012). This bias in the behavioral plan for the delay lever was conceptualized in the model as a transient prospective memory of the delayed reward that decayed over time and was modeled as the drift bias. The inclusion of this factor, slowed discounting, and provided an optimal fit to the Wistar experimental data. In addition, the inclusion of the drift bias reduced the number of steps required by the walk to find the delay boundary, bringing it closer to the number of steps required to find the immediate boundary (Fig. 6). This better modeled the experimental data, since the choice latencies in Wistar rats were similar for immediate and delay choices at the 8-s and 16-s delays, whereas delay choices were longer in the $\mathrm{P}$ rats. The ability of the drift bias to slow discounting and optimize the fit of the model to Wistar data suggests that a prospective memory, or behavioral plan, will facilitate the decision-making process. These data were further supported by follow-up experiments in which increasing the IRI length simultaneously decreased consistency and increased choice latencies in Wistar rats (Fig. 8). Collectively, these data underscore the importance of the ability to create a behavioral plan and hold it in prospective memory to delay reward and mitigate impulsive choices.

Addiction is characterized by an inability to delay immediate reward in light of future outcomes (de Wit, 2009). In this way, the alcoholic is constantly faced with a choice: Do I drink excessively to feel good now, or shall I abstain to feel good in the future? This choice requires that future consequences be conceptualized and integrated into a decision to drink or abstain. As we discussed, conceptualizing future rewards requires that their value be mapped to the context in order to form a prospective memory, which may be a critical intermediary to delay reward. Lack of planning, which involves prospective memory, has been described as one of five types of impulsivity (Whiteside \& Lynam, 2003), and a recent metaanalysis showed that lack of planning was one of the strongest predictors of alcohol dependence (Coskunpinar et al., 2013). In line with this view, nonplanning impulsiveness in recovering alcoholics has been shown to be associated with alterations in the activation and functional connectivity of brain regions thought to be critical for cognitive control, such as the dorsal anterior cingulate cortex (Jung et al., 2014).

The coordinated activity of multiple brain regions is necessary for tasks involving intertemporal decision-making such as those described here. Among these regions, alterations in neural processing in the hippocampus and frontal cortex are prime candidates for the differences observed between the P and Wistar rats in the present study (Peters \& Büchel, 2010, 2011). The hippocampus participates in the regulation of retrospective and prospective forms of episodic memory (Ferbinteanu \& Shapiro, 2003), with the former necessary for taking into account the consequences of previous reward choices, and the later necessary for forming plans based on the consequences of these previous choices. Given that lesions of the hippocampus in rats have been shown to increase impulsive choices (Cheung \& Cardinal, 2005; Mariano et al., 2009; Rawlins, Feldon, \& Butt, 1985), and that alterations have been reported in mRNA, protein, monoamines, and so forth, in this brain region in P rats (Edenberg et al., 2005; McBride \& Li, 1998; McBride, Murphy, Lumeng, \& Li, 1990; Stewart \& Li, 1997; Witzmann et al., 2003), functional differences between P and Wistar rats in the hippocampus may account in part for the differences in behavioral strategies and DD that we observed in this study. Regions of the frontal cortex are also known to be involved in the discounting of rewards (Cardinal, 2006), as well as in regulating other cognitive and executive functions (Dalley, Cardinal, \& Robbins, 2004). Interestingly, P rats display lower basal dopamine concentrations in the prefrontal cortex (PFC) than do Wistar rats (Engleman, Ingraham, McBride, Lumeng, \& Murphy, 2006). PFC dopamine has been found to facilitate the modification of choice biases during cost/benefit decision-making (St Onge, Ahn, Phillips, \& Floresco, 2012), suggesting that hypodompaminergia of the $\mathrm{PFC}$ in $\mathrm{P}$ rats may also be an important neurobiological mediator of DD in this rat population.

Collectively, these data identify heritable alterations in the decision-making process that lead to an impulsive phenotype and a heightened risk for excessive drinking. Moreover, these data highlight the need for further study of the neural processes that mediate impulsivity, and of the cognitive control mechanisms through which these processes are altered in individuals who are $\mathrm{FH}+$ for an AUD.

Author note This work was supported by NIAAA Grant Nos. AA022821 (to C.C.L.), AA023786 (C.C.L.), AA007611 (C.C.L.), and AA022268 (D.N.L.). This research was supported in part by Lilly Endowment, Inc., through its support for the Indiana University Pervasive Technology Institute, and in part by the Indiana METACyt Initiative. The Indiana METACyt Initiative at Indiana University is also supported in part by Lilly Endowment, Inc. We thank Dr. Brandon Oberlin for advice on the setup of our DD procedures. 


\section{References}

Acheson, A., Richard, D. M., Mathias, C. W., \& Dougherty, D. M. (2011). Adults with a family history of alcohol related problems are more impulsive on measures of response initiation and response inhibition. Drug and Alcohol Dependence, 117, 198-203.

Anokhin, A. P., Grant, J. D., Mulligan, R. C., \& Heath, A. C. (2014). The genetics of impulsivity: Evidence for the heritability of delay discounting. Biological Psychiatry, 77, 887-894. doi:10.1016/j. biopsych.2014.10.022

Beckwith, S. W., \& Czachowski, C. L. (2014). Increased delay discounting tracks with a high ethanol-seeking phenotype and subsequent ethanol seeking but not consumption. Alcoholism: Clinical and Experimental Research, 38, 2607-2614.

Beckwith, S. W., \& Czachowski, C. L. (2016). Alcohol-preferring P rats exhibit elevated motor impulsivity concomitant with operant responding and self-administration of alcohol. Alcoholism: Clinical and Experimental Research, 40, 1100-1110.

Bickel, W. K., Johnson, M. W., Koffarnus, M. N., MacKillop, J., \& Murphy, J. G. (2014). The behavioral economics of substance use disorders: Reinforcement pathologies and their repair. Annual Review of Clinical Psychology, 10, 641-677.

Bickel, W. K., Koffarnus, M. N., Moody, L., \& Wilson, A. G. (2014). The behavioral- and neuro-economic process of temporal discounting: A candidate behavioral marker of addiction. Neuropharmacology, 76(Pt. B), 518-527.

Bogacz, R., Wagenmakers, E.-J., Forstmann, B. U., \& Nieuwenhuis, S. (2010). The neural basis of the speed-accuracy tradeoff. Trends in Neurosciences, 33, 10-16. doi:10.1016/j.tins.2009.09.002

Braver, T. S. (2012). The variable nature of cognitive control: A dual mechanisms framework. Trends in Cognitive Sciences, 16, 106 113. doi:10.1016/j.tics.2011.12.010

Brown, S. D., \& Heathcote, A. (2008). The simplest complete model of choice reaction time: Linear ballistic accumulation. Cognitive Psychology, 57, 153-178. doi:10.1016/j.cogpsych.2007.12.002

Buckner, R. L., \& Carroll, D. C. (2007). Self-projection and the brain. Trends in Cognitive Sciences, 11, 49-57.

Cardinal, R. N. (2006). Neural systems implicated in delayed and probabilistic reinforcement. Neural Networks, 19, 1277-1301.

Cardinal, R. N., Daw, N., Robbins, T. W., \& Everitt, B. J. (2002). Local analysis of behaviour in the adjusting-delay task for assessing choice of delayed reinforcement. Neural Networks, 15, 617-634.

Cheung, T. H., \& Cardinal, R. N. (2005). Hippocampal lesions facilitate instrumental learning with delayed reinforcement but induce impulsive choice in rats. BMC Neuroscience, 6, 36.

Coskunpinar, A., Dir, A. L., \& Cyders, M. A. (2013). Multidimensionality in impulsivity and alcohol use: A metaanalysis using the UPPS model of impulsivity. Alcohol, Clinical and Experimental Research, 37, 1441-1450.

Crystal, J. D., \& Wilson, A. G. (2015). Prospective memory: A comparative perspective. Behavioural Processes, 112, 88-99. doi:10.1016 /j.beproc.2014.07.016

Dalley, J. W., Cardinal, R. N., \& Robbins, T. W. (2004). Prefrontal executive and cognitive functions in rodents: Neural and neurochemical substrates. Neuroscience \& Biobehavoral Reviews, 28, 771-784.

Dalley, J. W., Everitt, B. J., \& Robbins, T. W. (2011). Impulsivity, compulsivity, and top-down cognitive control. Neuron, 69, 680-694.

de Wit, H. (2009). Impulsivity as a determinant and consequence of drug use: A review of underlying processes. Addiction Biology, 14, 22-31.

Dick, D. M., \& Bierut, L. J. (2006). The genetics of alcohol dependence. Current Psychiatry Reports, 8, 151-157.

Dick, D. M., Smith, G., Olausson, P., Mitchell, S. H., Leeman, R. F., O'Malley, S. S., \& Sher, K. (2010). Understanding the construct of impulsivity and its relationship to alcohol use disorders. Addiction Biology, 15, 217-226.
Dougherty, D. M., Charles, N. E., Mathias, C. W., Ryan, S. R., Olvera, R. L., Liang, Y., \& Acheson, A. (2014). Delay discounting differentiates pre-adolescents at high and low risk for substance use disorders based on family history. Drug and Alcohol Dependence, 143, 105111.

Edenberg, H. J., Strother, W. N., McClintick, J. N., Tian, H., Stephens, M., Jerome, R. E., ... McBride, W. J. (2005). Gene expression in the hippocampus of inbred alcohol-preferring and -nonpreferring rats. Genes, Brain and Behavior, 4, 20-30.

Engleman, E. A., Ingraham, C. M., McBride, W. J., Lumeng, L., \& Murphy, J. M. (2006). Extracellular dopamine levels are lower in the medial prefrontal cortex of alcohol-preferring rats than for Wistar rats. Alcohol, 38, 5-12.

Enoch, M. A., \& Goldman, D. (2001). The genetics of alcoholism and alcohol abuse. Current Psychiatry Reports, 3, 144-151.

Ferbinteanu, J., \& Shapiro, M. L. (2003). Prospective and retrospective memory coding in the hippocampus. Neuron, 40, 1227-1239.

Green, L., \& Myerson, J. (2004). A discounting framework for choice with delayed and probabilistic rewards. Psychological Bulletin, 130, 769-792. doi:10.1037/0033-2909.130.5.769

Griffiths, A., Hill, R., Morgan, C., Rendell, P. G., Karimi, K., Wanagaratne, S., \& Curran, H. V. (2012). Prospective memory and future event simulation in individuals with alcohol dependence. Addiction, 107, 1809-1816.

Heffernan, T. M. (2008). The impact of excessive alcohol use on prospective memory: A brief review. Current Drug Abuse Reviews, 1, 36 41.

Howard, M. W., Fotedar, M. S., Datey, A. V., \& Hasselmo, M. E. (2005). The temporal context model in spatial navigation and relational learning: Toward a common explanation of medial temporal lobe function across domains. Psychological Review, 112, 75-116.

Jentsch, J. D., Ashenhurst, J. R., Cervantes, M. C., Groman, S. M., James, A. S., \& Pennington, Z. T. (2014). Dissecting impulsivity and its relationships to drug addictions. Annals of the New York Academy of Sciences, 1327, 1-26.

Jentsch, J. D., \& Pennington, Z. T. (2014). Reward, interrupted: Inhibitory control and its relevance to addictions. Neuropharmacology, 76(Pt. B), 479-486.

Johnson, E. O., van den Bree, M. B., Gupman, A. E., \& Pickens, R. W. (1998). Extension of a typology of alcohol dependence based on relative genetic and environmental loading. Alcoholism: Clinical and Experimental Research, 22, 1421-1429.

Jung, Y. C., Schulte, T., Muller-Oehring, E. M., Namkoong, K., Pfefferbaum, A., \& Sullivan, E. V. (2014). Compromised frontocerebellar circuitry contributes to nonplanning impulsivity in recovering alcoholics. Psychopharmacology, 231, 4443-4453.

Kim, S., \& Lee, D. (2011). Prefrontal cortex and impulsive decision making. Biological Psychiatry, 69, 1140-1146.

Kollins, S. H. (2003). Delay discounting is associated with substance use in college students. Addictive Behaviors, 28, 1167-1173.

Kurth-Nelson, Z., Bickel, W., \& Redish, A. D. (2012). A theoretical account of cognitive effects in delay discounting. European Journal of Neuroscience, 35, 1052-1064.

Leibowitz, N., Baum, B., Enden, G., \& Karniel, A. (2010). The exponential learning equation as a function of successful trials results in sigmoid performance. Journal of Mathematical Psychology, 54, 338-340.

Lejuez, C. W., Magidson, J. F., Mitchell, S. H., Sinha, R., Stevens, M. C., $\&$ de Wit, H. (2010). Behavioral and biological indicators of impulsivity in the development of alcohol use, problems, and disorders. Alcoholism: Clinical and Experimental Research, 34, 1334-1345.

MacKillop, J. (2013). Integrating behavioral economics and behavioral genetics: Delayed reward discounting as an endophenotype for addictive disorders. Journal of the Experimental Analysis of Behavior, 99, 14-31. 
MacKillop, J., Amlung, M. T., Few, L. R., Ray, L. A., Sweet, L. H., \& Munafò, M. R. (2011). Delayed reward discounting and addictive behavior: A meta-analysis. Psychopharmacology, 216, 305-321.

Mariano, T. Y., Bannerman, D. M., McHugh, S. B., Preston, T. J., Rudebeck, P. H., Rudebeck, S. R., ... Campbell, T. G. (2009). Impulsive choice in hippocampal but not orbitofrontal cortexlesioned rats on a nonspatial decision-making maze task. European Journal of Neuroscience, 30, 472-484.

Mazur, J. (1987). An adjusting procedure for studying delayed reinforcement. In M. L. Commons, J. E. Mazur, J. A. Nevin, \& H. Rachlin (Eds.), The effect of delay and of intervening events on reinforcement value (pp. 55-73). Hillsdale: Erlbaum.

McBride, W. J., \& Li, T. K. (1998). Animal models of alcoholism: Neurobiology of high alcohol-drinking behavior in rodents. Critical Reviews in Neurobiology, 12, 339-369.

McBride, W. J., Murphy, J. M., Lumeng, L., \& Li, T. K. (1990). Serotonin, dopamine and GABA involvement in alcohol drinking of selectively bred rats. Alcohol, 7, 199-205.

McBride, W. J., Rodd, Z. A., Bell, R. L., Lumeng, L., \& Li, T. K. (2014). The alcohol-preferring $(\mathrm{P})$ and high-alcohol-drinking (HAD) ratsAnimal models of alcoholism. Alcohol, 48, 209-215.

Mensink, G.-J. M., \& Raaijmakers, J. G. W. (1988). A model for interference and forgetting. Psychological Review, 95, 434-455.

Mitchell, S. H. (2011). The genetic basis of delay discounting and its genetic relationship to alcohol dependence. Behavioural Processes, 87, 10-17.

Murdock, B. B. (1997). Context and mediators in a theory of distributed associative memory (TODAM2). Psychological Review, 104, 839862. doi:10.1037/0033-295X.104.4.839

National Research Council. (2003). Guidelines for the care and use of mammals in neuroscience and behavioral research. Washington DC: National Academy of Sciences.

Oberlin, B. G., \& Grahame, N. J. (2009). High-alcohol preferring mice are more impulsive than low-alcohol preferring mice as measured in the delay discounting task. Alcoholism: Clinical and Experimental Research, 33, 1294-1303.

Perkel, J. K., Bentzley, B. S., Andrzejewski, M. E., \& Martinetti, M. P. (2015). Delay discounting for sucrose in alcohol-preferring and nonpreferring rats using a sipper tube within-sessions task. Alcoholism: Clinical and Experimental Research, 39, 232-238.

Peters, J., \& Büchel, C. (2010). Episodic future thinking reduces reward delay discounting through an enhancement of prefrontalmediotemporal interactions. Neuron, 66, 138-148. doi:10.1016/j. neuron.2010.03.026

Peters, J., \& Büchel, C. (2011). The neural mechanisms of inter-temporal decision-making: Understanding variability. Trends in Cognitive Sciences, 15, 227-239. doi:10.1016/j.tics.2011.03.002

Ratcliff, R., \& McKoon, G. (2008). The diffusion decision model: Theory and data for two-choice decision tasks. Neural Computation, 20, 873-922. doi:10.1162/neco.2008.12-06-420

Rawlins, J. N., Feldon, J., \& Butt, S. (1985). The effects of delaying reward on choice preference in rats with hippocampal or selective septal lesions. Behavioural Brain Research, 15, 191-203.

Rodriguez, C. A., Turner, B. M., \& McClure, S. M. (2014). Intertemporal choice as discounted value accumulation. PLOS ONE, 9, e90138. doi:10.1371/journal.pone.0090138

Roesch, M. R., Taylor, A. R., \& Schoenbaum, G. (2006). Encoding of time-discounted rewards in orbitofrontal cortex is independent of value representation. Neuron, 51, 509-520.
Rogers, R. D., Moeller, F. G., Swann, A. C., \& Clark, L. (2010). Recent research on impulsivity in individuals with drug use and mental health disorders: Implications for alcoholism. Alcoholism: Clinical and Experimental Research, 34, 1319-1333.

Romo, R., \& Salinas, E. (2001). Touch and go: Decision-making mechanisms in somatosensation. Annual Review of Neuroscience, 24, 107-137.

Shadlen, M. N., Britten, K. H., Newsome, W. T., \& Movshon, J. A. (1996). A computational analysis of the relationship between neuronal and behavioral responses to visual motion. Journal of Neuroscience, 16, 1486-1510.

St Onge, J. R., Ahn, S., Phillips, A. G., \& Floresco, S. B. (2012). Dynamic fluctuations in dopamine efflux in the prefrontal cortex and nucleus accumbens during risk-based decision making. Journal of Neuroscience, 32, 16880-16891.

Steiner, A. P., \& Redish, A. D. (2014). Behavioral and neurophysiological correlates of regret in rat decision-making on a neuroeconomic task. Nature Neuroscience, 17, 995-1002.

Stewart, R. B., \& Li, T. K. (1997). The neurobiology of alcoholism in genetically selected rat models. Alcohol Health and Research World, 21, 169-176.

Verbruggen, F., \& Logan, G. D. (2009). Models of response inhibition in the stop-signal and stop-change paradigms. Neuroscience \& Biobehavoral Reviews, 33, 647-661. doi:10.1016/j. neubiorev.2008.08.014

Verdejo-Garcia, A., Lawrence, A. J., \& Clark, L. (2008). Impulsivity as a vulnerability marker for substance-use disorders: Review of findings from high-risk research, problem gamblers and genetic association studies. Neuroscience \& Biobehavoral Reviews, 32, 777-810.

Walker, S. E., Pena-Oliver, Y., \& Stephens, D. N. (2011). Learning not to be impulsive: Disruption by experience of alcohol withdrawal. Psychopharmacology, 217, 433-442.

Wenger, G. R., \& Hall, C. J. (2010). Rats selectively bred for ethanol preference or nonpreference have altered working memory. Journal of Pharmacology and Experimental Therapauetics, 333, 430-436.

Whiteside, S. P., \& Lynam, D. R. (2003). Understanding the role of impulsivity and externalizing psychopathology in alcohol abuse: Application of the UPPS impulsive behavior scale. Experimental and Clinical Psychopharmacology, 11, 210-217.

Wilhelm, C. J., \& Mitchell, S. H. (2008). Rats bred for high alcohol drinking are more sensitive to delayed and probabilistic outcomes. Genes, Brain and Behavior, 7, 705-713.

Wilhelm, C. J., \& Mitchell, S. H. (2012). Acute ethanol does not always affect delay discounting in rats selected to prefer or avoid ethanol. Alcohol, 47, 518-524.

Wilhelm, C. J., Reeves, J. M., Phillips, T. J., \& Mitchell, S. H. (2007). Mouse lines selected for alcohol consumption differ on certain measures of impulsivity. Alcoholism: Clinical and Experimental Research, 31, 1839-1845.

Witzmann, F. A., Li, J., Strother, W. N., McBride, W. J., Hunter, L., Crabb, D. W., ... Li, T. K. (2003). Innate differences in protein expression in the nucleus accumbens and hippocampus of inbred alcohol-preferring and -nonpreferring rats. Proteomics, 3, 13351344.

Zeeb, F. D., Floresco, S. B., \& Winstanley, C. A. (2010). Contributions of the orbitofrontal cortex to impulsive choice: Interactions with basal levels of impulsivity, dopamine signalling, and reward-related cues. Psychopharmacology, 211, 87-98. 